\title{
«Ein Zeichen sind wir, deutungslos»
}

\author{
Vom verlorenen Ich zum Gedenken ans Sichverdanken
}

Was ist der Mensch? Diese in ihrer Allgemeinheit kaum zu beantwortende Frage erscheint zunächst nicht besonders geeignet für Festtagsgedanken zum Geburtstag eines passionierten Theologen. Nicht nur deshalb, weil eine Gott ignorierende Antwort, wie Martin Luther in seiner Disputatio de homine vorführt ${ }^{1}$, das Ziel des Menschseins verfehlt, sondern auch deshalb, weil sie bestenfalls die differentia specifica des Menschenwesens im Vergleich zu anderen Lebewesen ans Licht zu bringen vermag, ihr aber die Singularität des einzelnen Menschen entgeht. Auf nichts anderes jedoch als auf die Singularität eines unvergleichlichen Menschen verweisen Geburtstagsgrüsse - zumindest dann, wenn sie nicht gänzlich unpersönlich bleiben sollen. Sollen sie persönlich werden, legt sich eine andere Frage näher: Was zeichnet diesen in seiner Art ganz einzigartigen Menschen vor allen anderen Menschen aus?

Individuum est ineffabile. Diese alte Einsicht warnt davor, das Einzigartige in Worte fassen zu wollen. Warum aber sollte es unaussprechlich sein? Hebt unser Sprechen und Erkennen nicht gerade beim Einzelnen an, dem wir als Einzelnen begegnen und dessen Individualität wir je auf individuelle Weise wertschätzen? Auch wenn dies der Fall ist, trifft unser Erkennen und Sprechen dennoch nicht das Individuum als Einzelnes, sondern nur das, was von ihm bereits verallgemeinert ist. Wir können jemanden ansprechen und direkt zu ihm sagen: «Schön, dass Du da bist!» Doch damit ist noch nicht gesagt, weshalb wir dieses sagen. Gerade dann, wenn die Worte von Herzen kommen und durch reiche Begegnungen gesättigt sind, ist es umso schwieriger, das Singuläre zu benennen. Geschichten müsste man erzählen und dürfte nicht enden, wollte man alles erfassen, was diese

1 Vgl. M. Luther, Disputatio de homine, in: Martin Luther Studienausgabe, Bd. 5, hrsg. von H.-U. Delius, Berlin 1992, 126-133, These 32: «homo est iustificandus fide». Das Gerundivum bestimmt das Wesen des Menschen durch das, was an ihm geschieht. Das Sein des Menschen ist nicht ohne Gottes Handeln an ihm zu denken! Nach Luther ist das subiectum theologiae deshalb sowohl cognitio Dei wie auch cognitio hominis, nämlich der homo reus et perditus vor dem deus iustificans vel salvator (WA 40/ II, 327f).Vgl. G. Ebeling, Disputatio de homine (Lutherstudien 2), Bd. 1-3, Tübingen 1977-1989, zu These 32 vgl. Bd. 3, 27f, 404-409, 427f, 483ff. 
Begegnungen ausmacht. Wissen vom Einzelnen ist mehr als Wissen nur von einem Einzelnen, und Worte über einen Menschen werden Worte über andere Menschen. Das Einzigartige kann nicht ohne das Andersartige zur Sprache kommen, das Unvergleichliche nicht ohne Vergleichbares. Selbst wenn es gelingt, einen Einzelnen von Anderen abzugrenzen, geschieht es durch Worte, die vielfach verwendbar sind. Würden wir neue Worte für seine unvergleichliche Einzigartigkeit erfinden, wären dieselben dennoch nicht davor bewahrt, $\mathrm{zu}$ anderen Zeiten auf anderen Zungen dazu zu dienen, die Einzigartigkeit eines mit ihm verglichenen anderen Einzelnen zu bezeichnen.

\section{Nicht im Bilde}

Demnach ist die Frage nach dem Wesen dieses Menschen, d.h. eines bestimmten Menschen, nicht einfacher zu beantworten als die Frage nach dem Wesen des Menschen, d.h. aller Menschen. Die Bestimmung des Singulären scheint unerschöpflich, und die des Universalen strittig. So stellt sich die Frage, ob wir nicht überhaupt auf derlei Bestimmungsversuche verzichten sollten. Max Frisch verbindet diesen Verzicht mit dem Bilderverbot. Ein ihm gemässes «Menschenbildverbot» sieht er der Liebe aufgetragen:

«Das ist das Erregende, das Abenteuerliche, das eigentlich Spannende, daß wir mit den Menschen, die wir lieben, nicht fertigwerden: weil wir sie lieben; solang wir sie lieben. [...] Du sollst dir kein Bildnis machen, heißt es, von Gott. Es dürfte auch in diesem Sinn gelten: Gott als das Lebendige in jedem Menschen, das, was nicht erfaßbar ist. Es ist eine Versündigung, die wir, so wie sie an uns begangen wird, fast ohne Unterlaß wieder begehen - Ausgenommen wenn wir lieben.» ${ }^{2}$

Jeder Mensch ist mehr als das, was man von oder in ihm sieht. Er bewegt sich heraus aus dem Rahmen, innerhalb dessen sich jemand ein Bild von ihm machen kann. Doch ist es nicht leicht, sich kein Bild zu machen, denn auch der programmatische Versuch, auf Menschenbilder und eine annähernde Bestimmung des oder dieses Menschenwesens $\mathrm{zu}$ verzichten, enthält noch eine implizite Bestimmung, gleichsam das Negativ des Bildes: die bestimmte Unbestimmtheit bzw. Unbestimmbarkeit des Menschen. Friedrich Nietzsche nannte den Menschen dementsprechend nicht das animal

2 Vgl. M. Frisch, Vorstudien zu «Andorra», in: Tagebuch. 1946-1949, Frankfurt a.M. 1973, 31 und 37. 
rationale, sondern «das noch nicht festgestellte Tier» ${ }^{3}$. Der Mensch ist genau dadurch gekennzeichnet, dass ihm seine eigene Existenz und Essenz fraglich sind. Er ist zugleich das Subjekt und das Objekt einer möglichen Definition seiner selbst, doch bleibt er sich all seiner Definitionsbemühungen zum Trotz entzogen. Wie können wir jemals den Einzelnen in seinerTiefe ausloten, wie das uns Gemeinsame in seiner Breite festlegen, wie uns selbst verstehen in Höhen und Tiefen, Länge und Breite, Wirklichkeit und Möglichkeit?

\section{Erinnerung an das verlorene Ich}

Es sieht so aus, als seien wir uns selbst verloren gegangen - dergestalt, dass wir uns nicht finden, wo wir uns suchen. Oder dergestalt, dass wir uns anders bzw. als andere finden als wir glaubten, dass wir seien. «Es blieb die Erinnerung.» ${ }^{4}$ So kommentiert Hans Blumenberg unsere Lage und stellt die Frage nach der menschlichen Natur in einen memorialen Horizont. Der Mensch befindet sich auf dem Weg zu sich und findet sich allenfalls auf metaphorischen Umwegen - über die Requisiten, Bilder und Stichworte der Erinnerung. Aber kann er sich so wirklich wieder finden? Blumenberg selbst bezweifelt es und hält das Sich-wieder-Finden «bei aller Not, sich ein mögliches Selbstverständnis zu erzwingen» trotzdem für aussichtslos. ${ }^{5}$

Wäre Anthropologie nichts weiter als die Erinnerung daran, was es war, was wir wissen wollten, als wir fragten 'Was ist der Mensch? ${ }^{6}$, dann wäre sie nur 〈rückwärts〉 gerichtet, auf Erinnerung nicht nur derVergangenheit, sondern auf Erinnerung, die mit dem Menschen vergeht - oder schon verging. Das Präteritum des Zitats ist paradox, weil es einerseits die Erinnerung selbst in die Vergangenheit versetzt, andererseits aber ihr Bleiben behauptet. Nur das jedoch ist in der Tat geblieben, was bis heute verblieben ist. Wenn die Erinnerung nicht in die Gegenwart hinein reicht, ist die Vergangenheit vergessen, und mit ihr die damals noch unverwirklichten menschlichen Möglichkeiten. Gerade wenn die Vergangenheit auch in Erinnerung

3 Vgl. F. Nietzsche, Jenseits von Gut und Böse, III, 62.

${ }^{4}$ H. Blumenberg, Ein mögliches Selbstverständnis. Aus dem Nachlaß, Stuttgart 1997, 44. Ich danke Ulrik Houlind Rasmussen, durch dessen Vortragsmanuskript «Menschliche Erinnerung zwischen Wirklichkeit und Möglichkeit. H. Blumenbergs und P. Ricœurs Denken als nach-metaphysische Erinnerungsarbeit» ich auf das Zitat aufmerksam geworden bin.

5 Ebd., 45.

6 H. Blumenberg, Beschreibung des Menschen.Aus dem Nachlaß hrsg. von Manfred Sommer, Frankfurt a.M. 2006, 482f. 
dessen lebt, was hätte sein können und der Mensch nach dem sucht, was er noch nicht gewesen ist, sondern erst noch werden wird, wenn also die Vergangenheit auch eine Frage der Zukunft ist, dann genügt es nicht, die Frage nach der Natur oder dem Wesen des Menschen nur im Präteritum zu stellen. Die Frage nach dem einst begriffenen Menschenwesen greift zu kurz, denn der damalige Begriff (und das, was damals noch unbegriffen blieb) erscheint als solcher nur von dem her, was wir heute von uns denken. Was wir dachten, sehen wir im Nachhinein, wenn wir es nicht länger glauben. Deshalb stellt Blumenberg in seiner Beschreibung des Menschen der Frage «Was war es, was wir hatten wissen wollen?» zwei weitere Fragen an die Seite: "Was ist es, was wir wissen können? und: Was tun wir dort, wo wir aufWissen verzichtet haben oder noch verzichten müssen?» ${ }^{7}$

Dem Nichtwissen und der aporetischen Situation der IchVerlorenheit zum Trotz bleibt Blumenberg auf der Suche nach einem möglichenSelbstverständnis.Wo aber,wenn nichtin der Vergangenheit, in der wir uns befanden, verloren, und auch in Erinnerung nicht wieder finden, sollen wir uns suchen? Ironischerweise steht der Satz «Es blieb die Erinnerung» in einem Kapitel über «Selbstbeständigkeit», und zwar im Kontext einer Auslegung von Gottfried Benns Gedicht vom «Verlorenen Ich» (1943). Verloren gegangen war es im «Gewühl der Empfindungen». Wie kann es sich in Empfindungen verlieren, die doch an es selbst als empfindendes Ich gebunden sind?

Verloren gegangen in ihrem Gewühl scheint weniger das Ich als seine Einheit. Statt ungeteilt in eine bestimmte Richtung $\mathrm{zu}$ streben, scheint es mit sich selbst in Zwiespalt geraten und sich im Hin und Her einander widerstrebender Empfindungen verloren zu haben. Es blieb «nur die Memoria an das, was immerhin möglich gewesen, wenn auch nicht geblieben war.» ${ }^{8}$ Verharrend in Zwiespalt und Zweifel verliert das Ich vor allem seine eigene Zielstrebigkeit und mit ihr seine Handlungskraft. Wenn es die Möglichkeiten nicht ergreift, solange es sie hat, bleiben sie nicht seine Möglichkeiten. Mit seinen unverwirklichten Möglichkeiten verliert das Ich auch einen Teil seiner eigenen Wirklichkeit. Denn seine Wirklichkeit ist nicht nur kontingente Faktizität, sondern birgt ein Potential des Künftigen, das auch durch das bedingt ist, was es selbst gewählt und bestimmt hat. Im Wählen und Bestimmen legt es sich fest auf etwas Bestimmtes, Gewähltes, und im Wählen bestimmter Möglichkeiten schliesst es die Verwirklichung anderer Möglichkeiten aus. Durch seine eigene

\footnotetext{
Ebd., 482.

8 Dieses und das folgende Zitat stammen aus Blumenberg, Selbstverständnis, s. Anm. 4, 44.
} 
Selbstbestimmung wird das Ich daher nicht minder bestimmt als durch sein Sich-nicht-bestimmen-Können. $\mathrm{Ob}$ sich ihm verspielte Möglichkeiten noch einmal zuspielen oder nicht, dies liegt nicht in seinen eigenen Möglichkeiten. Aber kann es sich die verspielten Möglichkeiten nicht zumindest in dem Sinn saneignen`, dass es sich darüber klar wird, was ihm möglich war und was jetzt dagegen jenseits seiner Möglichkeiten liegt? Könnte es sich nicht gerade dadurch wieder finden, dass es sich begrenzt findet?

Auch wenn wir unsere Grenzen erkennen, stehen wir in Gefahr, uns erneut im Vergangenen zu verlieren - in dem, was wir einmal waren. "Wir leben im Maße von der Erinnerung, wie wir die aktuelle Chance, das Erinnerte noch einmal zu sein, verlieren.» Ist Erinnerung nur Rückwendung in das, was nicht mehr ist, führt sie zum Selbstverlust. «Verloren das Ich, unbenannt noch das stattdessen möglich Gewordene - ohne die Mitte, der sich alle neigten und dabei doch nur ein Diagramm der Einheit simulierten. ${ }^{9}$ Ein Neues, Anderes, wenngleich noch Unbenanntes, ist bereits ermöglicht, doch hat das Ich darin seine Mitte verloren. Die einst Einheit stiftende Mitte erweist sich nun als simuliert, von anderen fabuliert. Auch das Bild vom vergangenen Ich wird brüchig. Wie aber kann sich das Ich noch verstehen, wenn es sich nicht mehr sammeln kann? Wenn es die Diskontinuität zwischen Vergangenheit und Gegenwart nicht in vergegenwärtigter Vergangenheit und Zukunft, d.h. erinnernd und erhoffend überbrücken kann, verliert es die Verbindung zwischen Einst und Jetzt und Dann. Wenn es sich nicht aufs Neue wird, hat das Ich mit sich gebrochen, ist über sich zerbrochen. Bleibt es uneins mit sich, ohne zugleich mit sich zusammenzuwachsen, wird Identität zur Episode. Blumenberg stellt fest, dass jeder Mensch nur ein Leben hat, doch stellt er in Frage, ob dies eine Leben auch eine Identität einschliesst - und äussert die Befürchtung, dass die Unverbesserlichen womöglich die einzig wirklich «identischen` sind. ${ }^{10}$ Wer bereut, versucht das Vergangene zu reparieren und kehrt um. Die Selbigkeit des Lebens schliesst Selbstveränderung nicht aus. Im besten Falle findet sich das Ich in einer neuen Zeit auf einem anderen Standpunkt wieder: in seinem eigenen, gleichwohl erneuerten, veränderten Leben.

9 Ebd., 44f

10 Ebd., 39f 


\section{Mnemosyne: «Ein Zeichen sind wir, deutungs- los»}

Der einzige 'Zeit-Ort, an dem uns deutlich werden kann, wie wir waren und was aus uns geworden ist, ist die Gegenwart, in der wir uns deuten, verstehen oder verfehlen. Dass wir uns sogar in dieser gedeuteten Gegenwart verlieren können, so dass wir uns selbst nicht mehr recht zu deuten wissen, bezeugt Friedrich Hölderlins enigmatische Zeile aus einer Gedichtvariation, die mit Mnemosyne überschrieben ist: «Ein Zeichen sind wir, deutungslos «. ${ }^{11}$

Das poetische Ich spricht von 〈uns» - wer aber sind "wir»? Schliesst das 'Wir〉 die Leser ein, oder spricht das Ich im Namen einer Gruppe, die einer anderen gegenüber abzugrenzen ist? Da keine Abgrenzungsversuche unternommen werden und in derselben Strophe umfassend von den «Menschen» als den «Sterblichen» die Rede ist, während die beiden letzten Strophen die "Gestorbenen» erwähnen, ist davon auszugehen, dass das Gedicht eine allgemeine anthropologische Aussage macht und von uns Lebenden im Gegensatz zu den Toten spricht. Offen bleibt allerdings, ob jeder von uns ein Zeichen ist, oder ob wir in unserer Gesamtheit ein einziges Zeichen ausmachen.

Ein Zeichen ist in der Regel vieldeutig. Hier aber heisst es, es sei «deutungslos». Wohlgemerkt, es ist nicht etwa 〈bedeutungslos». Vielmehr entsteht der Anschein, als stehe seine Bedeutung bereits fest. Doch genau dadurch wird die Rede widersprüchlich. Denn die Bedeutung von etwas steht nur dann fest, wenn jemand ihm Bedeutung verliehen hat. Im vorliegenden Fall handelt es sich um eine Selbstzuschreibung, d.h. dieselbe Person, die sich und andere als ein Zeichen deutet, behauptet zugleich von diesem Zeichen, es sei «deutungslos». Im Unterschied zur «Undeutbarkeit» negiert die (Deutungslosigkeit» nicht nur das Resultat, sondern auch die Tätigkeit des Deutens. Das deutende Konstatieren der eigenen Deutungslosigkeit ist ein performativer Selbstwiderspruch und zudem eine Anfrage an den Zeichencharakter jenes Zeichens, das wir angeblich sind. Der Sinn des Verbums «deuten〉 oszilliert zwischen

\footnotetext{
11 Abgedruckt in R. Reuß, «[...]/ Die eigene Rede des andern»: Hölderlins Andenken und Mnemosyne, Basel/Frankfurt a.M. 1990, 347f. Reuß bietet die meiner Kenntnis nach sorgfältigste Interpretation (vgl. ebd., 359-363, 380-400), der ich in diesem Kapitel weitgehend folgen werde. Er datiert die Niederschrift des Gedichtes auf den Zeitraum zwischen 1802 und 1806, d.h. zwischen Hölderlins Rückkehr aus Bordeaux und der Einlieferung ins Autenriethsche Klinikum.
} 
〈auslegen, interpretieren〉 und «zeigen, weisen` - ein Zeichen aber, das auf nichts zeigt, ist widersinnig.

$\mathrm{Zu}$ jedem Zeichenprozess gehören nicht nur res und signum, eine bezeichnete Sache und ein Zeichen(träger), sondern auch ein Interpret. Im Falle einer Selbstbezeichnung fallen zumindest der Zeichenträger und das Bezeichnete zusammen. Sie sind identisch, könnte man meinen. Doch meint man dies, übersieht man, dass der sich als Zeichen bezeichnende Mensch sich ipso facto interpretiert und als Interpret seiner selbst sich in einem Auslegungsspielraum bewegt, der ihm gestattet, von sich Abstand zu nehmen, sich anders zu verstehen und künftig nicht mehr als Zeichen zu bezeichnen. Wie auch immer er sich bezeichnet, er benötigt für diesen Zeichenprozess nicht nur sich selbst als Bezeichnender, Bezeichneter und Sich-Deutender, sondern auch noch andere sprachliche Zeichen. Diese Zeichen sind im Unterschied zu ihm selbst nur Medien und Objekte, nicht aber zugleich Subjekte der Semiose. Damit ist jedoch nicht gesagt, dass die Semiose nur ein selbstreferentieller Prozess sei. Hölderlins Vers lässt offen, wofür und für wen wir ein Zeichen sind. Damit bleibt auch die Möglichkeit offen, dass wir als Zeichen von uns wegweisen, für etwas anderes stehen oder zumindest durch andere(s) als uns selbst gedeutet werden. ${ }^{12}$ Ein Zeichen liegt nicht einfach vor. Es wird willkürlich von jemandem als etwas genommen oder in einem Verweisungszusammenhang entdeckt, und dies Nehmen oder Entdecken eines Zeichens geschieht in einem sich fortgesetzt differenzierenden Deutungsprozess. Als ein Zeichen sind wir nicht nur etwas ‘an sich〉- dies gilt höchstens von unserer Materialität als Zeichenträger. Vor allem stehen wir 〈ür etwas anderes bzw. weisen auf dieses hin und bleiben deutbar durch andere Zeichen bzw. Zeichenbenutzer.

Die behauptete Deutungslosigkeit lässt verschiedene Deutungen $\mathrm{zu}$ - aber werden (wir) je eine Deutung erhalten? Unklar bleibt, ob gemeint ist, dass wir im Augenblick keine Selbstdeutung haben, ob wir grundsätzlich zur Deutung unfähig sind, oder ob uns keine Fremddeutung mitgegeben ist. Was wir sind, lässt sich offenbar nicht definitiv durch Deutung beantworten. Dass wir uns als uns deutende - und dennoch in gewissem Sinne «deutungslose - Zeichen deuten, scheint eher in eine Iteration, wenn nicht gar in einen unendlichen Regress von Deutungen und Deutungsdeutungen hineinzu-

12 An dieser Stelle stimme ich nicht mit Reuß, Die eigene Rede, s. Anm. 11, 382 überein, der die Materialität des Zeichenträgers in Vers 1 objekt- und interpretenlos absolut gesetzt sieht. Richtig ist allerdings, dass Bezeichnetes und Interpret unbestimmt bleiben. 
führen. Die vermeintliche (Deutungslosigkeit) kann als Ergebnis eines Deutungsprozesses auch ein Ausdruck der Ratlosigkeit sein: Ratlosigkeit angesichts einer unüberschaubaren oder gar unendlichen Vielfalt von Deutungen, in welcher eine autoritative oder endgültige Deutung unverfügbar bleibt.

Der Zusammenhang mit den beiden folgenden Versen zeigt, dass es um die Bestimmung des 〈ganzen Menschen〉 geht; was aber gesagt wird, kann dieser Intention nicht gerecht werden:

«Schmerzlos sind wir und haben fast

Die Sprache in der Fremde verloren.»

Während «deutungslos» primär auf den Menschen als vernünftigen verweist, verweist «schmerzlos» primär auf den Menschen als empfindenden, doch sind beide Dimensionen miteinander verbunden, denn das Bewusstsein, der Schmerzempfindung beraubt zu sein, setzt sowohl kognitive Leistungen wie auch affektives Angegangensein voraus. Der Übergang vom zweiten zum dritten Vers bezeugt das Widerfahrnis eines Entzugs wider Willen. Am Ende von Vers 2 ist im Fast-Haben der transitive Bezug auf etwas zu Habendes vorgezeichnet, aber der Bezug wird nicht eingelöst, sondern verliert sich abgründig an der Versgrenze. Das semantisch gehaltvolle Verbum "haben» wird in ein entleertes, nur noch den Perfektbezug ausdrükkendes Hilfsverb verwandelt. Die Rede vom Fast-Verlorenhaben der Sprache verweist auf ein nicht-intentionales Moment im Sprechen und auf eine Gefährdung, die das Menschsein selbst in Frage stellt.

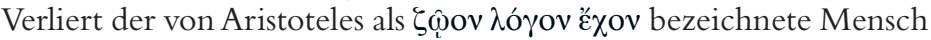
den Logos, die vernunftgebundene Sprache, steht das, was ihn von anderen Lebewesen unterscheidet, auf dem Spiel. Glückt es ihm nicht, sich das Befremdliche kommunikativ anzueignen, verliert er fast sein Eigenstes «in der Fremde», in der Entfremdung von sich selbst. Findet er sich wieder in der Sprache, verdankt er das SichFinden nicht sich selbst.

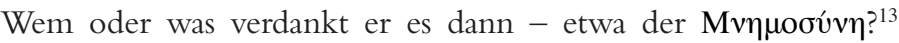
Mnemosyne, die Göttin des Gedächtnisses, gehört Hesiods Theogonie zufolge dem Geschlecht der Titanen an. Sie ist Tochter von Uranos und Gaia, Himmel und Erde, und die Mutter der Musen, die sie dem Zeus geboren hat. ${ }^{14}$ Die orphische Hymne an Mnemosyne sieht die Göttin als Verbindung zwischen vov̂s und $\psi v \chi \eta{ }^{15}$ Gibt die

\footnotetext{
${ }^{13}$ Das Folgende mit und im Gegensatz zu Reuß, Die eigene Rede, s. Anm. 11, 369$371,376,466,698 f$.

${ }^{14}$ Hesiod, Theogonie, 54, 915

15 Orpheus, Hymnus, 76 und 77.
} 
erste Strophe des Gedichts das allegorische Bild der Privation der Göttin und der verlorenen Verbindung zwischen Geist und Seele? Diese Deutung ist nicht auszuschliessen, doch bleibt der Bezug zwischen Titel und Text des Gedichtes mehrdeutig. Da es zu keinem Anruf kommt und das Gedicht nicht «An Mnemosyne〉 betitelt ist, bleibt fraglich, ob es sich überhaupt um eine Hymne handelt. Wäre Mnemosyne als Vokativ zu verstehen, verhallte der Ruf ohne Antwort und bliebe ein Bitten um die Gegenwart einer abwesenden, dem Text gegenüber extern bleibenden Göttin. Diese Deutung scheitert daran, dass die vierte Strophe «der Mnemosyne Stadt» erwähnt, und zwar in der Perspektive der dritten Person. Ist Mnemosyne für Hölderlin weniger als Göttin denn als Personifikation eines mentalen Phänomens von Belang? Dies trifft zu, sofern das Gedächtnis nicht auf ein menschliches Vermögen reduziert wird. Was uns einfällt und was wir vergessen, was uns gegeben wird und was wir davon behalten, ist - allen mnemotechnischen Anstrengungen zum Trotz - kaum kontrollierbar. Das Gedächtnis ist eine Gabe, über die wir nicht wie über ein Vermögen verfügen können. Entzieht sie sich uns, fehlen uns die Worte, und wir verlieren uns in der wortlosen Weite des Unbestimmten. An dieser Stelle zeigt sich ein Unterschied zwischen Erinnerung und Gedächtnis. Während Erinnerungen zunächst im Plural und mehr oder weniger unwillkürlich und ungeordnet auftreten, ist das Gedächtnis die konzentrierende Sammlung auf etwas Bestimmtes. Eher als er-innerndes Hineindenken ist Mnemosyne gedenkendes In-Eines-Denken.

Wenn sich nun der sprachliche Logos, die Vielfalt der Deutungen und das Eingedenken gleichermassen dem Gedächtnis verdanken, ist dies auch ein Hinweis darauf, wie sich das verlorene Ich wieder finden kann?

\section{Menschsein als Zeichen-Sein, Gedächtnis als An-dacht, Denken als Danken}

In seinen Freiburger Vorlesungen zur Frage Was heißt Denken? legt Martin Heidegger eine bemerkenswerte Auslegung von Hölderlins Versen vor. ${ }^{16}$

Bemerkenswert ist erstens die Weise, in der Menschsein als Zeichen-Sein verstanden wird. Ausgehend vom Gedanken, dass

\footnotetext{
${ }^{16}$ Vgl. M. Heidegger, Was heißt Denken?, Tübingen ${ }^{3} 1971$ (Vorlesungen im Wintersemester 1951-52 und im Sommersemester 1952 an der Universität Freiburg i.Br.), 6-9, $51 \mathrm{f}$.
} 
der Mensch der ist, der denken kann, kommt Heidegger auf dies "Bedenklichste» zu sprechen, "daß wir noch nicht denken» ${ }^{17}$ und fragt, woran das liegt. Er kommt zu dem Schluss, dass das, was uns zu denken gibt, keineswegs durch uns festgesetzt ist, sondern sich das zu-Denkende von je her in Abwendung von uns befindet. Doch kann uns das sich Entziehende wesentlicher angehen und in Anspruch nehmen als alles Anwesende, das uns trifft und betrifft. ${ }^{18}$ Dementsprechend expliziert Heidegger unser Zeichen-Sein als unser Verweisen auf dieses Sich-Entziehende: «auf dem Zug in dieses und somit zeigend in den Entzug, ist der Mensch allererst Mensch", seinem Wesen nach ein Zeichen, das deshalb ohne Deutung bleibt, weil es in das Sichentziehende zeigt ${ }^{19}$ und jenes noch nicht in die Sprache unseres Sprechens übersetzt, d.h. ebenfalls deutungslos ist. ${ }^{20}$ Wenngleich wir folglich nicht wissen, welches unser Wesen ist, ist es uns indirekt eröffnet - darin, dass wir geheissen sind, zu denken; aber unser Antworten auf die Frage (Was heißt Denken?) bleibt nichtsdestotrotz ein Unterwegsbleiben. ${ }^{21}$

Bemerkenswert ist zweitens die Weise, in der Mnemosyne eingeführt wird. Heidegger übersetzt den Namen der Titanide mit «die Gedächtnis` und erwähnt, dass Spiel und Musik, Tanz und Dichtung in ihren Schoss gehören. ${ }^{22}$ Mehrfach betont er, dass 〈Gedächtnis〉 anderes meint als nur die Fähigkeit, Vergangenes in der Vorstellung zu behalten - anderes als das Erinnerungsvermögen. ${ }^{23}$ Vielmehr nennt das Wort Heidegger zufolge das ganze Gemüt im Sinne der stetigen Versammlung bei dem, was sich allen Sinnen zuspricht, An-dacht im Sinne des unablässigen, gesammelten Bleibens beim Vergangenen, Gegenwärtigen und Zukünftigen gleichermassen. Als Sammlung des Gemüts ist «Gedächtnis` das Andenken an das zu-Denkende, das Gedenkbare und das Gedachte.

Bemerkenswert ist drittens die Verbindung von Gedächtnis, Denken und Danken. Heidegger führt aus, dass das Gemüt im Dank dessen gedenkt, was es hat und ist. Als Gedächtnis denkt es sich Jenem zu, dem es gehört, und deshalb ist der ursprüngliche Dank das Sich-Verdanken. ${ }^{24}$ Wem aber gehört das Gemüt, und wem denkt es sich zu? An anderer Stelle schreibt Heidegger nicht nur im Neutrum

\footnotetext{
17 Vgl. ebd., 1f, hier: 2.

8 Vgl. ebd., 3-5.

9 Ebd., 6.

${ }^{20}$ Vgl. ebd., 52

1 Vgl. ebd., 152, 164

22 Vgl. ebd., 6f.

23 Vgl. ebd., 7, 92, 97.

${ }^{24}$ Vgl. ebd., 93.
} 
vom Sich-Entziehenden, sondern stellt klar, dass es «der Gott» ist, der sich entzieht. ${ }^{25}$ Im Zusammenhang der Stelle bleibt jedoch offen, welcher Gott gemeint ist - Zeus, der biblische Gott, oder ein völlig anonymer Gott? Deutlich ist zumindest dies: Mnemosyne ist nicht gemeint. Fallen Gemüt und Gedächtnis ineins, sind sie beide auf der Seite des Menschen, doch dieser verdankt sich nicht sich selbst.

«Was wir zu verdanken haben, dies haben wir nicht aus uns. Es ist uns gegeben. Der Gaben empfangen wir viele und von mancherlei Art. Die höchste und eigentlich währende Gabe an uns bleibt jedoch unser Wesen, mit dem wir so begabt sind, daß wir aus dieser Gabe erst die sind, die wir sind. $»^{26}$

Für Heidegger ist diese Gabe, diese Mitgift unseres Wesens das Denken. Der höchste Dank dafür wäre, dass wir das Bedenklichste denken, und der tiefste Undank die Gedankenlosigkeit.

Das 〈Denken des Bedenklichsten〉 ist die Aufgabe jedes Menschen und insbesondere eine philosophische und theologische Herausforderung. Heideggers Deutung von Hölderlins Versen ist deshalb auch theologisch von Interesse, weil er das Zeichen-Sein des Menschen als ein Über-sich-hinaus-Zeigen interpretiert. Der Idee der ontologischen Suisuffizienz und der hermeneutischen Selbstgenügsamkeit des Menschen wird dadurch eine Absage erteilt. Das ist zu begrüssen. Aus Sicht der jüdisch-christlichen Tradition sind allerdings auch Rückfragen an Heidegger zu stellen: Ist andächtiges Denken tatsächlich die höchste Form des Danks an Gott? Wenn wir per definitionem in den Entzug des sich entziehenden Gottes Zeigende sind, bleiben wir dann noch Menschen, wenn Gott selbst sich auf uns beziehen und sich zeigen sollte? Anders gefragt: Verbaut sich Heidegger nicht die Möglichkeit, eine wechselseitig offenbarende Begegnung zwischen Gott und Mensch zu denken, wenn er unser Wesen exklusiv in Entsprechung zur Entzogenheit Gottes definiert? Es bleibt jedenfalls fraglich, ob sich das verlorene Ich im Zug auf das Sich-Entziehende zu finden vermag und jemals seine Aufgabe erfüllen wird.

25 Ebd., 7.

26 Ebd., 94. 


\section{Zeichen unserer selbst - Zeichen des Anderen}

Unter der Überschrift «MAN, A SIGN» entfaltet auch Charles Sanders Peirce den Gedanken, dass Menschsein Zeichen-Sein ist. ${ }^{27}$ Sein Ausgangspunkt ist die These «mind is a sign ${ }^{28}$, um dann den Unterschied zwischen Mensch und Wort herauszustellen:Der Mensch ist (sich seiner selbst und anderer Menschen und Dinge) bewusst, ein Wort ist es nicht. Menschliches Bewusstsein hängt nach Peirce von einem «animal body» ab, d.h. es ist rückgekoppelt an die Körperlichkeit und Materialität des Menschen-Zeichens. Wie die Worte erhalten auch die Menschen mehr und mehr Bedeutungszuwachs im Laufe der Zeit. Da Denken ohne Worte oder andere Symbole unmöglich ist, geht Peirce sogar so weit, nicht nur den Menschen wortschöpfende und bedeutungsgebende Funktionen zuzusprechen, sondern auch den Worten eine Lehrerfunktion. Die Menschen und die Worte unterweisen einander wechselseitig. ${ }^{29}$ Der Mensch kommt dabei sowohl als Zeichenmacher wie auch als Zeichenbenutzer und als durch Zeichen Gebildeter in den Blick, während die (angeblich bewusstlosen!) Worte in Peirces Beschreibung beinahe wie Personen agieren, sich dem Menschen zuwenden und mit ihm sprechen. Die hyperbolisch personalisierende Redeweise birgt eine nicht unproblematische Äquivokation des Zeichenbegriffs. Dies wird besonders deutlich in der Behauptung, dass der Mensch das Zeichen benutzt, das er ist. Jeder Gedanke sei ein Zeichen und das Leben ein Zug der Gedanken, was wiederum zeige, dass der Mensch ein Zeichen sei. Zweifellos involviert das menschliche Leben Zeichenprozesse, aber rechtfertigt dies die Gleichsetzung des Zeichenbenutzers mit den verwendeten Zeichen? Ist meine Sprache wirklich die Summe meiner selbst, und bin ich identisch mit meinen Gedanken $?^{30}$

Der Mensch als sembodied mind (notabene: nicht nur als Leib) ist nach Peirce der Beginn der Deutungen sowie ein Zeichen seiner

\footnotetext{
${ }^{27}$ Vgl. Ch. S. Peirce, "Questions Concerning Certain Faculties Claimed for Man» und «Some Consequences of Four Incapacities», in: Collected Papers of Charles Sanders Peirce. Volume V: Pragmatism and Pragmaticism and Volume VI: Scientific Metaphysics, hrsg. von Ch. Hartshorne/P.Weiss, Cambridge, Mass. 1960, 135-155, 156-189. Die hier interessierenden Ausführungen finden sich in der Schlusspassage ( $\mathbb{S} 5$ ) der beiden zusammengehörenden Essays von 1868, d.h. in den Abschnitten 310-316 auf den Seiten 185-189. Im Folgenden zitiere ich nach dem Schema «CP Bandnummer: Abschnitt).

${ }^{28}$ Ebd., CP 5: 313

${ }^{29}$ Ebd.: «men and words reciprocally educate each other.»

${ }^{30}$ Ebd., CP 5: 314: «Thus my language is the sum total of myself; for the man is the

thought.»
} 
selbst, bedeutungsvoll in sich. Zugleich bedeutet er mehr als er an und für sich ist. Er weist über sich hinaus, doch wohin weist er? Peirce bestimmt die Identität des Menschen als Konsistenz zwischen Denken und Handeln. ${ }^{31}$ Unser Zeichengebrauch beeinflusst die Modalität unseres Zeichen-Seins. Dabei verweist das eine Zeichen auf das andere. Peirces Definition des Zeichens als «something which stands to somebody for something in some respect or capacity « ${ }^{32}$ spricht von einem Verweisungszusammenhang, und diesen expliziert er nicht solipsistisch, sondern intersubjektiv. Wie das Sein eines Gedankens von nachfolgenden Gedanken abhängig ist, ${ }^{33}$ so kann kein Individuum unabhängig von einer Deutungsgemeinschaft fortexistieren. ${ }^{34}$ Das eine Zeichen ist nur im Zusammenhang mit einem anderen zu deuten, und die Bedeutung des einen Menschen erscheint nur in dem, was er für einen anderen bedeutet. Wir leben und bewegen uns in Welten des Deutens und Bedeutens. Aber wissen wir, wer oder was wir sind, jeder in seiner mehr oder weniger mit anderen geteilten Welt? Oder bleiben wir uns selbst ein Rätsel? Peirce scheint Letzteres anzunehmen. Abschliessend zitiert er die vielsagenden Verse:

«...proud man,

Most ignorant of what he's most assured,

His glassy essence. $\aleph^{35}$

Der Stolz heisst uns absehen oder uns absetzen von anderen. Stolz suggeriert ein Wissen um das Eigenste. Doch selbst dies ist durchsichtig auf andere. Wer nach seinem Wesen sucht, findet nie nur sich allein, wenn er sich findet. Sich-Wiederfinden heisst Sich-beianderen-Finden. Menschsein ist Zusammen-Sein: Sich-Bestimmen im Bestimmtsein durch die Nächsten. Als Zeichen seiner selbst ist der Mensch zugleich ein Zeichen des Anderen - und dies nicht nur für sich.

\footnotetext{
31 Vgl. CP 5: 315.

32 CP 2: 228.

${ }^{33} \mathrm{Vgl}$. CP 5: 316: «thought is what it is, only by virtue of its addressing a future thought $[\ldots]$ In this way, the existence of thought now depends on what is to be hereafter; so that it has only a potential existence, dependent on the future of the community."

${ }^{34}$ Vgl. CP 5: 317: «The individual man, since his separate existence is manifested only by ignorance and error, so far as he is anything apart from his fellows, and from what he and they are to be, is only a negation."

35 Ebd., Quelle ungenannt.
} 


\section{Zwischen den Zeichen}

Die hermeneutische Problematik des Zeichens ist eng mit seiner ontologischen Problematik verknüpft. Das poetische Ich «ist nicht ohne das, was es sagt . Spricht es nicht, so ist es nicht. Ein einzelnes Zeichen kann nur zwischen anderen Zeichen 〈sein〉. Das 〈Ich〉 als Zeichen besagt etwas im Zusammenhang mit einem 〈Du〉, mit 〈hm〉, 〈hr > und «ihnen - in konkreter Kommunikation. Es liegt seinen Äusserungen nicht zugrunde, sondern steht und fällt mit ihnen. Es verweist auf andere, ohne die es nicht sagen könnte, was es besagt. Steht der Zeichenerfinder nicht mehr 〈hinter〉 seinen Zeichen und verleiht ihnen kein künftiger Zeichenverwender seine Stimme, so bezahlen sie mit ihrem literarischen «Leben».

Menschen wirken wie Zeichen. Sie drücken sich aus, sie appellieren und sie stellen etwas dar. Aber sind sie nichts anderes als Zeichen unter Zeichen? Sind Menschen Zeichen, so setzen sie sich selbst in Szene, werden aber auch von anderen benutzt. Als Zeichen sind Menschen Instrumente und Effekte des Gesprächs: Sie führen ein Gespräch miteinander und sie werden durch das Gespräch, das sie selbst sind, mit allem, was geschieht. Es bleibt der Verdacht, dass ein Zeichen, das sich selbst ins Gespräch bringen und zum Gesprächsgegenstand machen kann, das nicht nur sprechend ist, sondern spricht, das sich selbst versteht und nach sich sucht - dass solch ein Zeichen mehr ist als ein buchstäbliches Zeichen.

Aber worin besteht dies «Mehr〉 des Menschen-Zeichens? Es wird nicht nur gebraucht, sondern braucht sich selbst, um sich und andere $\mathrm{zu}$ sehen. Sein Stand- und Blickpunkt zwischen Zeichen ist ihm nicht gleichgültig, genauso wenig wie das, was andere sehen. Der Mensch ist ein Zeichen mit Augen und Ohren, Mund und Nase, Armen und Beinen, Leib und Seele, Aussen und Innen. Es bekümmert sich um seine Existenz und ist besorgt um die prekäre Existenz des Anderen. Es isst und trinkt, lebt und stirbt. Es ringt nach Atem und nach Worten. Dies Zeichen ist kein Zeichen wie es im Buche steht. Was im Buche steht, ist nur sein Repräsentant. Es selbst jedoch bewegt sich weit über den in Büchern geschaffenen Raum hinaus, es steht und geht, fühlt und sieht sich um. Zwar lassen sich auch Gefühle als Zeichenprozesse verstehen, doch sind dies Prozesse, die sich primär zwischen Personen abspielen und von den Personen zu den Zeichen und zurück zu den Personen führen. Personen sind auf andere Weise zwischen den Zeichen als Zeichen zwischen Personen sind. 


\section{Nicht im Bilde, aber in Gottes Gedenken}

Nachdem der Unterschied zwischen sprachlichen Zeichen und sich der Sprache bedienenden Personen geklärt ist, kommen wir zurück zur Frage, in welchem Sinne Menschen nicht nur wie Zeichen wirken, sondern selbst als Zeichen zu verstehen sind. Die Antwort fällt verschieden aus, je nachdem, welche Zeichenart man in den Blick nimmt.

Wird der Mensch zum Beispiel als Index verstanden, verweist er gestisch oder kausal auf andere(s). Relevant sind hier raumzeitliche Relationen sowie die Rollen, die er persönlich einnimmt. Wird der Mensch als Symbol verstanden, verweist er arbiträr auf andere(s). Relevant sind hier seine sprachlichen Zeichen und Signalcodes, von denen er seiner Individualität gemäss Gebrauch macht. Wird der Mensch als Ikon verstanden, verweist er bildhaft-analog auf andere(s). Relevant sind hier jene Züge, in denen er dem, worauf er verweist, entspricht. Die biblische Vorstellung vom Menschen als imago Dei baut auf letztgenannte Zeichenart. Worin genau besteht seine Gottebenbildlichkeit - etwa im aufrechten Gang, in der Vernünftigkeit, Verantwortlichkeit oder in der Herrscherstellung des Menschen? Die Antworten auf diese Frage sind nicht minder spekulativ wie jene auf die Frage nach seinem Wesen.

Mit Max Frischs Vorbehalt im Hintergrund seien nun nicht noch weitere inhaltliche Antworten herausgestellt, sondern vielmehr die Problematik aller Antwortversuche.Wenn es stimmt, dass Menschsein und Menschwerden ein Zeichenereignis ist, dann ist der Mensch als Bild Gottes sich nur über den Umweg der Semiose als Mensch zugänglich, genau wie Gott selbst nicht direkt, sondern nur zeichenvermittelt von anderem unterscheidbar als Gott zugänglich ist bzw. sich in ZZeichen und Wundern〉 offenbart. Da der Zeichenprozess unabschliessbar bleibt, solange Menschen leben und mit Gott verbunden sind, macht es keinen Sinn, eine abschliessende Bestimmung des oder dieses Menschen vorlegen zu wollen.

Wenn es ausserdem stimmt, dass der Anfang der Schöpfung nicht temporal, sondern semiotisch als Anfang der Zeichen $\mathrm{zu}$ fassen ist, da es ohne Zeichenprozesse keine Zeitprozesse gibt, und dass die Vollendung der Schöpfung nicht als Ende der Zeichen und Zeiten, sondern als hermeneutisches Ende der Zweideutigkeit der Zeichen zu denken ist, da ein Ende der Zeichen dieVernichtung der Schöpfung wäre, ${ }^{36}$ dann dürfen wir weder damit rechnen, dass wir

${ }^{36}$ Vgl. I.U. Dalferth, Zeit der Zeichen.Vom Anfang der Zeichen und dem Ende der Zeichen, in: ders., Gedeutete Gegenwart. Zur Wahrnehmung Gottes in den Erfah- 
ohne Deutungen auskommen, noch dass unsere eigenen Deutungen die einzig richtigen sind, sondern können uns getrost darauf verlassen, dass Gott selbst seine Geschöpfe auf sein heilvolles Wirken hin transparent zu machen weiss. Soll auch das verlorene Ich auf ewig selig sein, dann bleibt es in Gottes Gedenken selbst dann, wenn es nicht über sich im Bilde ist:

"[...] was ist der Mensch, dass du seiner gedenkst,

und des Menschen Kind, dass du dich seiner annimmst?»

(Psalm 8,5)

Was der Mensch ist, wird nicht beantwortet. Es wird erneut erfragt. Doch was auch immer er ist und wie er sich denkt, ob er überhaupt an sich denkt oder sich vergisst, Gott denkt an ihn und nimmt sich seiner an. Aus den Worten des Psalms spricht ein Sich-Wundern. Wir wissen nicht, wer wir sind, wir erinnern uns nicht, wer wir waren, und wir ahnen nicht, was aus uns wird - und dennoch dürfen wir leben und uns unseres Lebens freuen. Das ist eindeutig ein Grund zum Dank!

Und ein Grund, Geburtstag zu feiern.

לחיים !

- Dr. Claudia Welz ist Postdoctoral Research Fellow am Center for Subjectivity Research der University of Copenhagen.

rungen der Zeit, Tübingen 1997, $227 \mathrm{f}$ 